\title{
Approach to Choose of Optimal Number of Turns in Planar Spiral Coils for Systems of Wireless Power Transmission
}

\author{
Konstantin Krestovnikov, Ekaterina Cherskikh*, Aleksandr Bykov \\ Laboratory of Autonomous Robotic Systems, \\ St. Petersburg Federal Research Center of the Russian Academy of Sciences (SPC RAS), \\ St. Petersburg Institute for Informatics and Automation of the Russian Academy of Sciences, \\ $3914^{\text {th }}$ Line, 199178, St. Petersburg, Russia \\ cherskikh.e@iias.spb.su
}

\begin{abstract}
Correct choice of coil parameters for resonant circuits in inductive power transmission systems is a relevant problem, as it significantly influences the efficiency and transmitted power in the systems and provides for optimization of these parameters. This paper presents a methodology of calculation of geometrical and electrical parameters and approach to choose the optimal number of turns in planar coils used in the wireless power transmission (WPT) system with parallel resonant circuit. Formulas are derived for calculation of active resistance and inductance of the coil, normalized to the specified design parameters of the coil. Connection is made between the design and electrical parameters of the coil, which allows choosing the optimal number of turns according to different criteria and guard conditions. The examples of practical use of the chosen approach with transmitting and receiving coils of WPT system are presented. The obtained results show that efficiency and transmitted power in the system are higher when using the coils with the calculated number of turns. The proposed approach may be used in selection of optimal design of loop coils in systems with fixed frequency, and in systems, whose operational frequency depends on the parameters of the resonant circuit.
\end{abstract}

Index Terms-Coils; Inductive power transmission; Optimization; Wireless power transmission.

\section{INTRODUCTION}

Wireless power transmission with inductively coupled coils is employed in such domains as robotics, automotive industry, healthcare, etc. [1], [2]. Wireless dock stations embedded in asphalt covering enable battery charging for electric cars during they are stopped or waiting, what increases the endurance of vehicles [3], [4]. Currently, in autonomous robotic devices, battery cells are most frequently used for power supply, which are charged in contact and contactless manner [5], [6]. Wireless power transmission provides for greater autonomy of mobile robots. In swarm robotics, individual robots need to interrupt the task execution and return to dock station to recharge the battery [7]. Wireless power transmission system (WPT) allows to simplify this process and to reduce human involvement in it. Coils of different shapes and sizes

Manuscript received 16 May, 2020; accepted 18 September, 2020. can be applied in such inductive coupling. A common solution involves planar spiral coils with and without core. Planar coils have significant diameter/thickness ratio, what positively influences the coupling factor between them. Because they are slim, they can be used in restricted space settings.

The main parts of WPT system are the receiving coil and the transmitting one, which define the efficiency and transmitted power of the whole system. Coil parameter optimization is performed to boost these metrics; hence, it poses a relevant problem. Our scientific interest is focused mostly on application of WPT systems in robotics. However, let us consider several existing solutions in various fields to get a broader overview of the topic.

One of the applied domains for utilization of planar spiral coils in wireless power transmission are the implanted medical devices. Coils can be integrated into living tissues on hard and soft printed boards. To obtain required inductance, the optimum number of turns should be defined, as well as turn spacing and coil size. For example, the authors in [8] propose a pair of printed rectangular spiral coils intended for use in implanted neuroprosthetics. They propose a design methodology aimed to select the optimal geometrical parameters of the coils. When designing the cortical visual prosthetics, the following parameters of the device were subject to constraints: inner and outer diameters, coil spacing, working frequency, conductor dimensions, nominal load value, thickness, and dielectric permeability of the substrate. The following parameters were optimized: coil size, fill factor, wire diameter, and gap between the coils of the receiving and the transmitting coils. Optimizations were performed in iterative manner, until the efficiency reached the maximum value for the selected working frequency. Because the working frequency of the system influences the efficiency and optimal geometry of the printed coils, the authors fabricated two prototypes with working frequencies of $1 \mathrm{MHz}$ and $5 \mathrm{MHz}$ with effective power transmission of $41.2 \%$ and $85.8 \%$ respectively by $10 \mathrm{~mm}$ distance. The disadvantage of this solution consists in maintaining fixed distance between the coils, because when putting WPT system in practice, mutual positioning of 
coils can influence the performance efficiency and transmitted power.

As in the previous paper, the coils presented in [9] are intended for implanted devices. The authors consider an assembly of resonant circuits with four squared coils. They provide a methodology for optimizing parameters of the coils to achieve high efficiency of WPT system performance considering the following constraints: load resistance, gap between coils, and frequency. The authors conducted experiments with biological tissue and presented the experimental results with different variations of planar coils. Using four coils, the efficiency is increased due to high Qfactor. Both round and square coil shapes are analysed and optimized to ensure maximum efficiency at certain spacing between the receiving and transmitting coils. The analysis revealed that the round coils show lower Q-factor and power transmission efficiency, than the square ones. At the same time, the developed methodology has a specific field of application and corresponding limitations on scaling.

The methodology of coil design and optimization of multiband wireless link for neuroprosthetic implantable devices is presented in [10]. For power transmission and data transfer, as well as for reverse telemetry, three separate signals and coil/antenna pairs are used. The power is transmitted at frequency of $13.56 \mathrm{MHz}$ with a pair of printed spiral coils. The authors proposed two designs of coils in respect to potential application domain. They studied how the wire diameter and number of turns (from 0 to 10) influences the coupling coefficient between the coils. If the coil positions relative to each other remain stable, then the pair of perpendicularly positioned coils is proposed, wound down the perimeter of printed spiral power coils. By variable spacing between the coils, accompanied by axial deflection, authors suggest using a pair of planar coils laid out in 8-knot pattern and being in the same plane as the planar power coils. A configuration is proposed where power transmission is effected by varying distance between the transmitting and receiving parts, whereby the WPT system performance drops significantly when the distance between the coils increases. The proposed methodology is intended for calculating the range of parameters needed for neuroprosthetic applications.

Thus, coil device optimization is performed to achieve the required inductance in implanted medical devices, which being inserted into living tissues, are strictly constrained in size and weight. When using the coils in medical devices [11], [12], it is necessary to define the efficiency and transmitted power by different possible layouts of transmitting and receiving coils relative to each other, because the coils are not stationary and may be displaced in the process of power transmission. However, any choice of coil parameters depends on the intended application [13], [14], [15] and still requires more unified techniques.

For example, the transportation sector requires significantly more powerful WPT systems than the prototypes discussed above. Let us consider several solutions in this field. The authors in [16] presented a WPT system for electric bicycles with power of $100 \mathrm{~W}$. Bearing on the required value of mutual inductance, optimization of turn number was performed, and the value of 9 turns was chosen for both receiving and transmitting coils. When selecting the number of turns, the authors also considered the requirements that the coil must be lightweight, compact, and cheap. WPT system optimization based on mutual inductance value is a suboptimal solution, because by varying distance between the receiving and transmitting coils, the mutual inductance also changes. However, this approach is impractical in robotics, because axial deflection and coil spacing alterations are possible, which render the whole system inefficient.

A WPT system for electric cars is presented in [17]. The authors show an analytical model of the system, in which the influence of system dimensions on mutual inductance is studied. Based on the analytical model, the coil dimension optimization is performed, where the working frequency of the coils is $10 \mathrm{kHz}$ [18]. Further, the system performance efficiency before and after the optimizations is calculated. It was revealed, that to achieve high efficiency, the radius of the receiving coil should be close to the radius of the transmitting coil, if the distance between two coils is less than the radius of the transmitting coil. If this distance exceeds the radius of the transmitting coil, as well as when the coils are displaced relative to each other, the radius of the receiving coil should be increased proportionally to the distance between the coils. The efficiency of the optimized system is $19 \%$ higher than in the non-optimized design by coil spacing of $30 \mathrm{~cm}$ and transmitting coil radius of $20 \mathrm{~cm}$. The maximum efficiency of the optimized WPT system is $85 \%$ and it is achieved with air gap of $20 \mathrm{~cm}$, whereas the efficiency of the non-optimized system is $67 \%$. The proposed optimization method based on the finite element method can be applied to rectangle-shaped coils.

In indoor robotics application, when the working area of robots is defined and limited, the following solution can be implemented. The transmitting part of the charging device is mounted under the surface, on which the robot works, what effectively detaches it from the stationary dock station. This setup allows proceeding with the task execution while recharging the battery. Such a WPT system, based on inductive coupling, is presented in [19]. The authors performed structural optimization of transmitting and receiving coils and studied the influence of turn number in the coil on coupling factor. The optimization objective consisted in the increase of allowable mutual displacement of the coils and system performance increase. During optimization, the performance of single-layer and doublelayer coils, each containing 7 turns, was performed. It was revealed that double-layer coils are preferable compared to single-layer ones. The performance efficiency of the optimized prototype is $50 \%$ in the allowable range of coil displacement (from $0 \mathrm{~mm}$ to $100 \mathrm{~mm}$ ). The output power by the values of air gap and frequency given above allows employing the proposed WPT system for charging of mobile robots.

Prior to optimization, theoretical calculations and empirical comparisons of different proposed designs of planar coils in practice are performed. The key coil parameters subject to optimization are size, wire diameter, number of turns, copper volume, and fill factor. During optimization, the finite element method is often employed 
with the aid of specialized software. In the papers considered above, the authors optimize the geometrical parameters and the number of turns in the coil in context of mutual inductance parameters, coupling coefficient, and power transmission efficiency.

The proposed methodologies do not allow respecting and considering all the coil characteristics and their indirect parameters, which influence the efficiency and transmitted power in WPT systems, as every methodology is aimed to improve only a certain characteristic. The calculation of direct and relevant indirect parameters of spiral coils, as well as selection of the optimal solution in the context of the specified constraints, provide for increase of efficiency and transmitted power, as well as reduce size and weight of the finished system. The aim of this paper consists in the development of a calculation methodology and an approach to selecting the optimal number of coil turns considering the specified constraints for planar helical coils utilized in WPT systems as loop coils. The proposed solutions can be applied to select the optimal coil model depending on the target parameters: loop coil size, wire diameter, operational frequency, allowable current, power losses, and other indirect parameters. The distinctive feature of the proposed approach is that it is not intended to optimize loop coil metrics relative to the known mutual layout of inductively coupled coils or related metrics, what is relevant in terms of real-world WPT system operation.

\section{CAlculation OF PARAMETERS OF THE LOOP COIL AND SELECTION OF TURN NUMBER}

WPT system performance efficiency heavily depends on correct parameter selection for loop coils in receiving and transmitting loop. Loop coil optimization is a multi-criterion problem due to coupled parameters indirectly influencing each other. Planar spiral loop coil (Fig. 1) can be characterized in terms of its design features by the following parameters: number of turns $N$, coil pitch $d$, and initial winding radius $r$.

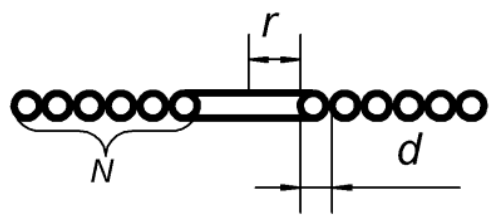

Fig. 1. Design parameters of a planar spiral coil.

The parameter of power transmission efficiency directly depends on the coupling coefficient between the receiving and the transmitting coil, which is calculated upon the mutual inductance between the coils [20]

$$
k_{\text {coup }}=\frac{M_{12}}{\sqrt{L_{1} L_{2}}}=\sqrt{\frac{\Phi_{M 1} \Phi_{M 2}}{\Phi_{11} \Phi_{22}}},
$$

where $M_{12}$ is mutual inductance; $L_{1}, L_{2}$ are inductances; $\Phi_{M 1}, \Phi_{M 2}$ are magnetic fluxes of mutual inductance; $\Phi_{11}$, $\Phi_{22}$ are magnetic fluxes of self-inductance in the transmitting and the receiving coil, respectively.

WPT system utilization assumes variable air gap and axial displacements between the coils. Consequently, the coupling coefficient is not a constant value, but according to (1), it decreases with increase of power transmission distance, because such increase causes drop in inductive coupling $M_{12}$. Coupling coefficient shows which part of the magnetic flux, induced by one coil, causes electromotive force $(\mathrm{EMF})$ in the second coil

$$
\varepsilon_{2}=-k_{\text {coup }} \frac{d \Phi}{d t}
$$

Hence, as follows from (2), to increase the transmitted power and performance efficiency of the system as a whole, generally it is required to amplify either the coupling coefficient between the coils, what is feasible only if the parameters of mutual layout of receiving and transmitting coils can be changed, or the magnetic flux, which induces EMF in the receiving loop. In real-world application of WPT systems in robotic devices, precise mutual layout [21] of coils by energy transfer and repeated achievement of it poses a difficult task. In the situation described above, this approach has no practical advantages over the contact methods. Hence, selection of the optimal kind of loop coil should not be based on mutual inductance parameter and its maximization.

According to the Biot-Savart-Laplace law, the value of the magnetic inductance vector caused by any elementary current in every point of space is proportional to this current. The magnetic flux is proportional to the value of magnetic inductance $B$, which in turn is proportional to the current $I$ in the conductor. This leads to conclusion that $\Phi$ $I$ and the inductance value $L(3)$ is the proportionality factor

$$
\Phi=L I
$$

It follows from (3) that it is possible to increase the magnetic flux generated by the transmitting coil boosting the current that flows through it or boosting its inductance value. Thereby, the parameters $L$ and $I_{m}$ of the coil in the LC loop working at the resonant frequency are coupled according to (4)

$$
I_{m}=\frac{U_{m}}{\sqrt{(2 \pi f L)^{2}+R_{c o n}^{2}}},
$$

where $U_{m}$ is amplitude of alternating voltage fed to the resonant circuit, $f$ is working frequency of the loop coil, and $R_{\text {con }}$ is active resistance of coil conductor.

Altering of turn number in coil modifies its inductance, and consequently its reactance. In addition, with number of turns changes the length of the conductor and its active resistance. In presence of such connections, any parameters of the coil must be calculated depending on the number of turns. To that end, it is necessary to express all the estimated relationships through the geometric parameters of the coil.

Electric parameters of the coil are its inductance and active resistance values. The value of active resistance of the uniform conductor with uniform cross-section is calculated according to (5) 


$$
R_{c o n}=\frac{p l_{c o n}}{S}
$$

where $\rho$ is specific electrical resistance of the conductor stuff; $l_{\text {con }}$ is conductor length; $S$ is cross-sectional area of the conductor.

The length of the conductor in a spiral planar coil depending on the number of turns is calculated according to (6)

$$
l_{\text {con }}=2 \pi\left(N r+d\left(0.5 N^{2}-0.5 N\right)\right)
$$

The active resistance of the conductor in the spiral coil was calculated by (5) and (6).

Calculation of inductance value of the planar spiral coil can be performed with decent accuracy according to the empirical Wheeler formula [22], [23] normalized to the metrical units and geometrical parameters of the coil given above

$$
L=\frac{\mu_{0}}{4 \pi} \frac{1000}{2.54} \frac{(2 r+N d)^{2} N^{2}}{32 r+60 N d},
$$

where $\mu_{0}$ is magnetic constant.

Upon the current amplitude value $I_{m}(4)$, losses $P_{d}$ are calculated, which are caused by active resistance of the loop coil

$$
P_{d}=\left(\frac{I_{m}}{\sqrt{2}}\right)^{2} R_{c o n}
$$

To perform coil comparison and choose the optimal number of turns, a parameter must be introduced that can be correlated with the loss parameter $P_{d}$ and that characterizes the amount of energy of the magnetic field being accumulated in the loop coil per unit of time

$$
P_{m}=\left(\frac{I_{m}}{\sqrt{2}}\right)^{2} L f .
$$

The $\gamma$ parameter allows contrasting the coils with different number of turns more vividly. The $\gamma$ is the ratio of loss in the loop coil to the energy of magnetic field accumulated in the coil per unit of time

$$
\gamma=\frac{P_{d}}{P_{m}} .
$$

The $\gamma$ coefficient is the Q-factor of the loop coil expressed through power on the given frequency. The $\gamma$ values less than 1 show that coil losses exceed the magnetic field energy being accumulated in the coil per unit of time. Increasing the number of turns, the loss in coil drops, because inductance and reactance in it grow, and consequently the idle current value decreases.

The approach to turn number selection assumes tabular calculations, as by such data representation it is most convenient to compare and contrast the estimated coil parameters. The initial parameters for calculations are: $U_{m}-$ amplitude of alternating voltage fed to the resonant circuit, $f$
- working frequency of the loop coil, $r$ - initial radius of the winding, $d-$ coil pitch, $\rho-$ specific resistance of the conductor stuff, $S$ - conductor cross-section, and $j-$ permissible current density in the conductor.

This paper extensively studies the possibilities of selection of the optimal number of turns $N^{*}$ in the coil by predefined initial radius and coil pitch. Though the formulas given above can be used for selection of optimal coil parameters by variable pitch or initial radius, or working frequency of the system.

As an example, a permissible current value for the conductor was specified, as a limiting condition in the presented approach to selection of optimal turn number

$$
I_{m}^{p}: \boldsymbol{N}=\left\{N \mid\left(N_{1} \leq N \leq N_{2}\right) \wedge\left(I \leq I_{m}^{p}\right)\right\} \in \boldsymbol{R}^{n} .
$$

This parameter is calculated according to permissible current density $j$ and specified cross-section $S$ of the conductor

$$
I_{m}^{p}=j^{p} S
$$

The objective function is presented by the actual current in the coil conductor: $I: \boldsymbol{N} \rightarrow \boldsymbol{R}^{n}$. Therefore, to ensure the maximum transmitted power, $I(N) \longrightarrow N_{N \in N} \max$.

If the objective of the coil parameter selection is to minimize the weight and the dimensions of the coil, then the constraints concern coil size or resonant circuit capacitance. If a self-oscillator is used in the transmitting part of the WPT system, with frequency-setting LC circuit, the selection of the optimal coil with fixed capacitance of resonant circuit and variable working frequency, which depends on the inductance of the loop coil, may be of particular interest.

For selection of optimal number of turns of loop coil by $f$ = const , the range of possible turn values $\left[N_{1}, N_{2}\right]$ is set. For every $N$ value using the presented formulas, the geometrical $\left(l_{\text {con }}\right)$ and electrical $\left(R_{\text {con }}, L\right)$ coil parameters are calculated. Further, the actual current value $I=I_{m} / \sqrt{ } 2$ in the conductor is defined, depending on which the optimal number of turns в $N^{*}$ is chosen, so that $I\left(N^{*}\right)=\max I(N)$.

By $f=v a r$, first for each $N$, geometrical and electrical parameters of the coil are calculated, then the working frequency is determined according to (13)

$$
f=\frac{1}{\sqrt{L C}} .
$$

Further, similarly as by $f=$ const, $I$ and $N^{*}$ are defined. By specified constraints of coil dimensions, its outer diameter is calculated according to the formula $D=2(r+$ $N d$ ), and the number of the turns is chosen so that $D_{\max } \leq D$.

\section{PARAMETER CALCULATION AND OPTIMAL TURN NUMBER SELECTION CASE}

The evaluation of the developed approach was performed by comparison of theoretical calculation results with the experimental data. In the Table I, the initial parameters are given for selection of optimal turn number for the receiving 
and transmitting coils of low-power WPT system for further employment in swarm robotic system to redistribute energy among swarm agents [24]-[26]. Circuit and design solutions for the receiving [27], [28] and transmitting parts of WPT system are presented in our previous research, where the WPT system of moderate power is considered [29], [30].

TABLE I. INITIAL PARAMETERS FOR THE CALCULATIONS

\begin{tabular}{|c|c|c|c|}
\hline Parameter & Value & Parameter & Value \\
\hline$U_{m}$ & $15.7 \mathrm{~V}$ & $\rho$ & $0.0171 \mathrm{Ohm} \cdot \mathrm{mm}^{2}$ \\
\hline$f$ & $44 \mathrm{kHz}$ & $S$ & $0.374 \mathrm{~mm}^{2}$ \\
\hline$r$ & $19 \mathrm{~mm}$ & $j^{p}$ & $6 \mathrm{~A} / \mathrm{mm}^{2}$ \\
\hline$d$ & $0.74 \mathrm{~mm}$ & & \\
\hline
\end{tabular}

Table II shows the results of theoretical calculation of parameters for the coil with turn number range from 1 to 20 by constant working frequency.

TABLE II. CALCULATION RESULTS BY $f=$ const

\begin{tabular}{|c|c|c|c|c|c|c|}
\hline $\begin{array}{c}\boldsymbol{N}, \\
\text { turn }\end{array}$ & $\begin{array}{c}\boldsymbol{R}_{\text {con }}, \\
\mathbf{O h m}\end{array}$ & $\boldsymbol{L}, \boldsymbol{\mu H}$ & $\boldsymbol{I}, \mathbf{A}$ & $\boldsymbol{P}_{\boldsymbol{d}}, \mathbf{W}$ & $\boldsymbol{P}_{\boldsymbol{m}}, \mathbf{W}$ & $\boldsymbol{\gamma}$ \\
\hline 1 & 0.0055 & 0.09 & 433.43 & 1025.41 & 1497.24 & 1.46 \\
\hline 2 & 0.0111 & 0.35 & 113.31 & 142.90 & 398.03 & 2.79 \\
\hline 3 & 0.0170 & 0.77 & 51.79 & 45.63 & 182.53 & 4.00 \\
\hline 4 & 0.0231 & 1.35 & 29.81 & 20.53 & 105.18 & 5.12 \\
\hline 5 & 0.0294 & 2.06 & 19.46 & 11.14 & 68.70 & 6.17 \\
\hline 6 & 0.0359 & 2.92 & 13.75 & 6.79 & 48.56 & 7.15 \\
\hline 7 & 0.0427 & 3.91 & 10.25 & 4.49 & 36.23 & 8.07 \\
\hline 8 & 0.0496 & 5.05 & 7.96 & 3.14 & 28.11 & 8.95 \\
\hline 9 & 0.0568 & 6.31 & 6.36 & 2.30 & 22.48 & 9.78 \\
\hline 10 & 0.0641 & 7.71 & 5.21 & 1.74 & 18.40 & 10.58 \\
\hline 11 & 0.0717 & 9.25 & 4.34 & 1.35 & 15.34 & 11.35 \\
\hline 12 & 0.0795 & 10.92 & 3.68 & 1.08 & 13.00 & 12.08 \\
\hline 13 & 0.0875 & 12.73 & 3.15 & 0.87 & 11.15 & 12.80 \\
\hline 14 & 0.0958 & 14.68 & 2.74 & 0.72 & 9.67 & 13.49 \\
\hline 15 & 0.1042 & 16.76 & 2.40 & 0.60 & 8.47 & 14.16 \\
\hline 16 & 0.1128 & 18.99 & 2.12 & 0.50 & 7.48 & 14.81 \\
\hline 17 & 0.1217 & 21.36 & 1.88 & 0.43 & 6.65 & 15.44 \\
\hline 18 & 0.1308 & 23.87 & 1.68 & 0.37 & 5.95 & 16.07 \\
\hline 19 & 0.1401 & 26.54 & 1.51 & 0.32 & 5.35 & 16.67 \\
\hline 20 & 0.1496 & 29.35 & 1.37 & 0.28 & 4.84 & 17.27 \\
\hline
\end{tabular}

Based on (12), for the given initial parameters, the permissible current value in the conductor $I_{m}^{p}$ is $2.24 \mathrm{~A}$. Consequently, feasible designs are the coil with the number of turns ranging from 15 to 20 . Loop coil with 15 turns has the actual current value of $2.4 \mathrm{~A}$, what exceeds the calculated value of $I_{m}^{p}$ approximately by $7 \%$. Hence, it is possible to use this design. Considering the specified current constraint, the coil with 15 turns is the best fit.

Calculation results for the case with variable working frequency are shown in Table III. The calculation is performed for the capacitance of the resonant circuit $C=$ $0.47 \mu F$

According to the Table III, allowable variants in context of the constrained current value in coil conductor are the ones in the range from 12 to 20 turns. Based on the criteria of maximum transmitted power and energy transmission distance, the optimal variant is the coil with 12 turns. Comparing the coils with optimal number of turns by $f=$ const and $C=$ const, we conclude that in the latter case the
Q-factor is higher, whereas the lost power is 1.4 times lower. In the case of $C=$ const, the $P_{m}$ is also lower. It equals to $8.14 \mathrm{~W}$, which is less than in the 15-turn coil on $44 \mathrm{kHz}$ frequency approximately by $3.9 \%$. It is also worth noting, that by loss level of $0.6 \mathrm{~W}$ in the 15-turn coil in calculations with $C=$ const, such a loss value corresponds to the 8-turn coil. In this case, magnetic field energy accumulated in the coil per unit of time will be higher, specifically, $P_{m}=11.98 \mathrm{~W}$.

\begin{tabular}{|c|c|c|c|c|c|}
\hline $\begin{array}{l}N, \\
\text { turn }\end{array}$ & $f, \mathbf{k H z}$ & $I, \mathrm{~A}$ & $\boldsymbol{P}_{d}, \mathbf{W}$ & $\boldsymbol{P}_{m}, \mathbf{W}$ & $\gamma$ \\
\hline 1 & 771.41 & 25.30 & 3.49 & 89.45 & 25.60 \\
\hline 2 & 391.14 & 12.83 & 1.83 & 45.35 & 24.76 \\
\hline 3 & 263.99 & 8.66 & 1.28 & 30.61 & 24.00 \\
\hline 4 & 200.16 & 6.56 & 1.00 & 23.21 & 23.30 \\
\hline 5 & 161.67 & 5.30 & 0.83 & 18.74 & 22.66 \\
\hline 6 & 135.87 & 4.46 & 0.71 & 15.75 & 22.07 \\
\hline 7 & 117.33 & 3.85 & 0.63 & 13.60 & 21.53 \\
\hline 8 & 103.35 & 3.39 & 0.57 & 11.98 & 21.02 \\
\hline 9 & 92.40 & 3.03 & 0.52 & 10.71 & 20.55 \\
\hline 10 & 83.59 & 2.74 & 0.48 & 9.69 & 20.10 \\
\hline 11 & 76.33 & 2.50 & 0.45 & 8.85 & 19.69 \\
\hline 12 & 70.25 & 2.30 & 0.42 & 8.14 & 19.29 \\
\hline 13 & 65.07 & 2.13 & 0.40 & 7.54 & 18.92 \\
\hline 14 & 60.60 & 1.99 & 0.38 & 7.03 & 18.57 \\
\hline 15 & 56.70 & 1.86 & 0.36 & 6.57 & 18.24 \\
\hline 16 & 53.27 & 1.75 & 0.34 & 6.18 & 17.93 \\
\hline 17 & 50.23 & 1.65 & 0.33 & 5.82 & 17.63 \\
\hline 18 & 47.51 & 1.56 & 0.32 & 5.51 & 17.35 \\
\hline 19 & 45.07 & 1.48 & 0.31 & 5.22 & 17.08 \\
\hline 20 & 42.85 & 1.41 & 0.30 & 4.97 & 16.82 \\
\hline
\end{tabular}

\section{EXPERIMENTAL Testing OF THE PROPOSED APPROACH}

For the estimation of efficiency of the proposed approach, the real-world experiments were performed with the selected optimal variants of loop coils comprising the WPT system, which are further compared with a coil having an ad-hoc number of turns from the possible range (Table II and Table III). It is necessary to bear in mind that the objective is to maximize the transmitted power and efficiency of the system with the specified current limit in the loop coil. The performed tests are intended to support only the validity of the developed approach to selection of turn number in coils, but not the accuracy of calculation of numerical dependent parameters. To test the proposed approach, three kinds of coils were made with 12, 15, and 19 turns, respectively. In the first experiment, the consumed power of the transmitting part of the system and output power of the receiving part of the system were tested with fixed power transmission distance. At the first stage of the experiment, two coils with 15 and 19 turns were compared within a WPT resonant circuit with operational frequency of $44 \mathrm{kHz}$. The results of this stage of the experiment are presented in Fig. 2 as a function of energy transfer efficiency from transmitted power. The coils were in close proximity during the experiment.

As follows from the presented curve, when using the 15turn coil, the energy transfer efficiency increases in the whole range of the transmitted power. The maximum 
transmitted power was $8.77 \mathrm{~W}$ for the 15-turn coil and $6.55 \mathrm{~W}$ for the 19-turn coil, energy transfer efficiency reached $54 \%$ and $48.6 \%$, respectively. The calculated value $P_{m}$ is $3.4 \%$ lower than the experimentally obtained maximum value of transmitted power. This discrepancy is caused by the influence of inductive coupling between the coils on the working frequency and parameter divergence in actual elements of the resonant circuit.

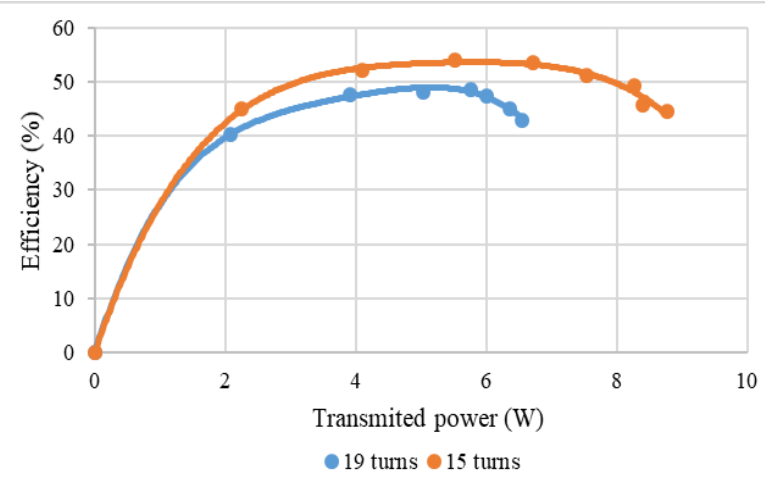

Fig. 2. Dependency of the system efficiency from the transmitted power for the coils with 19 and 15 turns.

In the second stage of the experiment, the coils with 12 and 19 turns were compared in the WPT resonant circuit with fixed capacitance $C=0.47 \mu F$. The obtained dependencies are shown in Fig. 3.

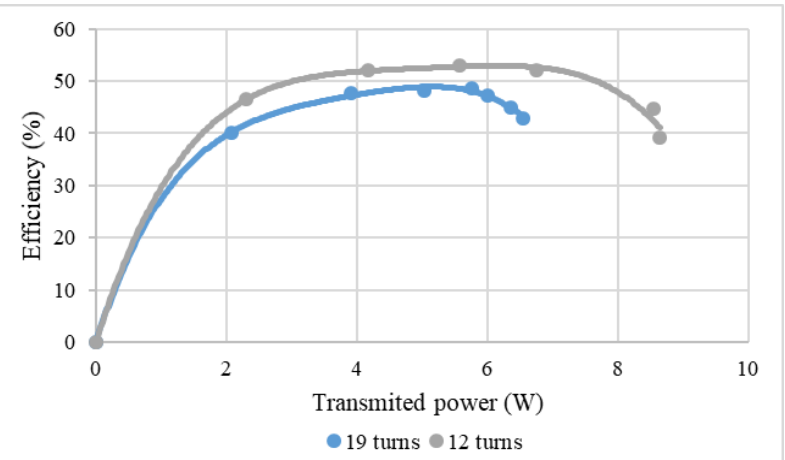

Fig. 3. Dependency of the system efficiency from the transmitted power for the coils with 19 and 12 turns.

As follows from the graphical dependencies, the efficiency of wireless energy transfer system in the whole range of transmitted power is higher for the 12-turn coil than for the 19-turn coil. The system reaches maximum efficiency of $53 \%$ with transmitted power of $5.58 \mathrm{~W}$. The maximum transmitted power with 12-turn coil was $8.64 \mathrm{~W}$.

The second experiment-included measurement of consumed power in transmitting and receiving parts of the system with fixed load resistance connected to the receiving part and variable distance between the receiving and transmitting coils. The dependencies obtained for the efficiency and transmitted power of the system from the energy transfer distance for three kinds of coils are shown in Fig. 4 and Fig. 5.

When the distance between the coils increases, the performance efficiency and transmitted power of WPT system drops because of decrease of mutual inductance value. Power transmission efficiency with 12-turn and 15turn coils is higher than with 19-turn coil in the whole energy transfer range. The maximum power transmission efficiency is achieved at distances less than $25 \mathrm{~mm}$ with a 15-turn coil. The average difference value in power transmission efficiency between 19-turn and 15-turn coils is $7.5 \%$.

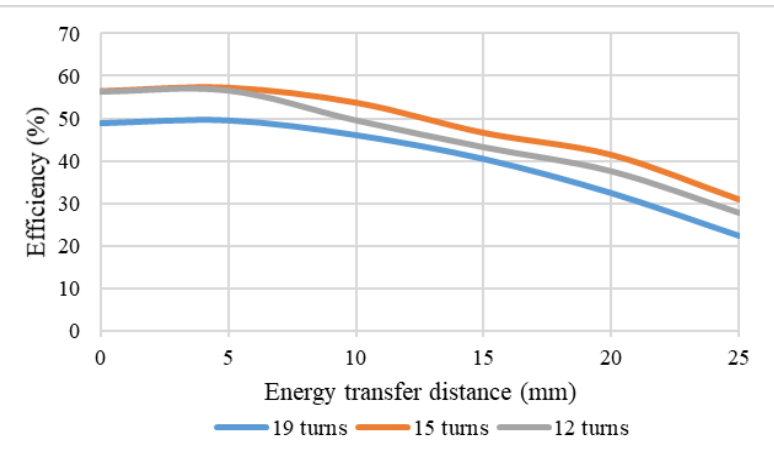

Fig. 4. Dependency of system efficiency from power transmission distance for coils with 19,15 , and 12 turns.

As follows from the curve in Fig. 5, the transmitted power value in the whole range of distances is higher in optimal coil designs. For the 15-turn coil, the transmitted power in the range of distances less than $25 \mathrm{~mm}$ is by $79.6 \%$ higher than in 19-turn coil, on average. In 12-turn coil design, the average value of difference in transmitted power was $30.4 \%$.

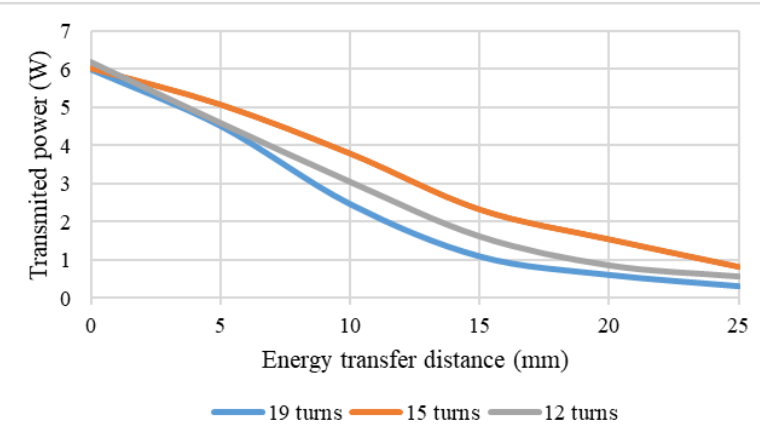

Fig. 5. Dependency of the transmitted power of the system from energy transfer distance for the coils with 19, 15, and 12 turns.

The curves of dependencies for efficiency and transmitted power for the coils with 12 and 15 turns are similar in shape and values, what conforms to theoretical calculations. The curves of the transmitted power and efficiency for the 12turn coil are lower down the y-axis than the respective curves for 15-turn coil, what indirectly confirms the validity of theoretical calculations presented above. The experimentally obtained parameters of efficiency and transmitted power are higher when using theoretically calculated optimal coil designs.

The experimentally obtained results confirm the theoretically calculated data based on the developed methodology, as well the correctness of the developed approach to selection of turn number for a planar spiral coil. The presented methodology enables calculations to choose the optimal design of loop coil in WPT systems intended to maximize one of the system parameters: the transmitted power and efficiency, allowable dimensions, operating frequency of the system, etc. The proposed approach, in contrast to other research, does not require acting upon predefined parameters of coupling coefficient or mutual 
inductance, and therefore it does not constrain coil layout when the system is put in practice, what is particularly relevant in mobile robotic devices.

\section{CONCLUSIONS}

The calculation methodology presented in this paper, as well as the approach to selection of the optimal number of turns for the loop coil, allow finding the best solution respecting indirect parameters by specified criteria and constraints. Tabular calculations allow conveniently representing the coil parameters, comparing and contrasting different designs. The paper presents a case of coil parameter calculation by fixed frequency and capacitance of the resonant circuit. From the calculated designs, the ones are chosen that meet the criteria of maximum transmitted power respecting the limit of permissible current in the coil conductor. Experimental test was performed via comparison of the suboptimal coil designs chosen upon calculation results, with the suboptimal one. Based on the obtained values, graphical dependencies are drawn for system efficiency and transmitted power from the energy transfer distance. The obtained results show that the system efficiency and transmitted power are higher when using the coils selected according to the proposed approach. Transmitted power at $10 \mathrm{~mm}$ distance when using the optimal coil design is $54 \%$ higher in calculations with the fixed working frequency of the system and $23.5 \%$ higher with fixed capacitance value for the resonant circuit. The increase in power transmission efficiency is $7.5 \%$ and $3.4 \%$, respectively. When the coils are in close proximity, the optimal coil design allows increasing the transmitted power by $33.8 \%$ for a 15 -turn coil and by $31.9 \%$ for the 12-turn coil, maximum efficiency difference compared to 19-turn coil amounts to $5.9 \%$ and $5 \%$ respectively by load current of $1.2 \mathrm{~A}$. The obtained experimental data confirm correctness of the presented calculation methodology and of the developed approach to the selection of turn number for planar spiral coils. Application of the proposed methodology for calculation of coil parameters in wireless power supply systems for autonomous sensors can significantly influence the development of cyberphysical systems [31], [32].

\section{CONFLICTS OF INTEREST}

The authors declare that they have no conflicts of interest.

\section{REFERENCES}

[1] F. Pellitteri, "Wireless charging systems for electric vehicle batteries", Ph.D. dissertation, University of Palermo, 2016.

[2] V. Boscaino, F. Pellitteri, L. Rosa, and G. Capponi, "Wireless battery chargers for portable applications: Design and test of a highefficiency power receiver", IET Power Electronics, vol. 6, no. 1, pp 20-29, 2013. DOI: 10.1049/iet-pel.2012.0272.

[3] F. Musavi and W. Eberle, "Overview of wireless power transfer technologies for electric vehicle battery charging”, IET Power Electronics, vol. 7, no. 1, pp. 60-66, 2014. DOI: 10.1049/ietpel.2013.0047.

[4] S. Li, and Ch. Ch. Mi, "Wireless power transfer for electric vehicle applications", IEEE Journal of Emerging and Selected Topics in Power Electronics, vol. 3, no. 1, pp. 4-17, 2015. DOI: 10.1109/JESTPE.2014.2319453.

[5] J. Guo, R. Zheng, X. Yu, A. Wei, and B. Yang, "Autonomous underwater vehicle docking system based on wired transmission", in
Proc. of WRC Symposium on Advanced Robotics and Automation (WRC SARA), 2018, pp. 1-6. DOI: 10.1109/wrc-sara.2018.8584153.

[6] D.-j. Li, Y.-h. Chen, J.-g. Shi, and C.-j. Yang, "Autonomous underwater vehicle docking system for cabled ocean observatory network", Ocean Engineering, vol. 109, pp. 127-134, 2015. DOI 10.1016/j.oceaneng.2015.08.029.

[7] I. V. Bychkov, M. Yu. Kenzin, and N. N. Maksimkin, "Two-level evolutionary approach to persistent surveillance for multiple underwater vehicles with energy constraints", SPIIRAS Proceedings, vol. 2, no. 18, pp. 267-301, 2019. DOI: 10.15622/sp.18.2.267-301.

[8] U.-M. Jow and M. Ghovanloo, "Design and optimization of printed spiral coils for efficient transcutaneous inductive power transmission", IEEE Transactions on Biomedical Circuits and Systems, vol. 1, no. 3, pp. 193-202, 2007. DOI: 10.1109/TBCAS.2007.913130.

[9] S. R. Khan and G. Choi, "Analysis and optimization of four-coil planar magnetically coupled printed spiral resonators", Sensors, vol. 16, no. 8, p. 1219, 2016. DOI: 10.3390/s16081219.

[10] U.-M. Jow and M. Ghovanloo, "Optimization of data coils in a multiband wireless link for neuroprosthetic implantable devices", IEEE Transactions on Biomedical Circuits and Systems, vol. 4, no. 5 , pp. 301-310, 2010. DOI: 10.1109/tbcas.2010.2049491.

[11] S. Rao and J.-C. Chiao, "Body electric: Wireless power transfer for implant applications", IEEE Microwave Magazine, vol. 16, no. 2, pp. 54-64, 2015. DOI: 10.1109/MMM.2014.2377586.

[12] P. Li and R. Bashirullah, "A wireless power interface for rechargeable battery operated medical implants", IEEE Transactions on Circuits and Systems II: Express Briefs, vol. 54, no. 10, pp. 912-916, 2007. DOI: 10.1109/TCSII.2007.901613.

[13] A. Beyzavi and N.-T. Nguyen, "Modeling and optimization of planar microcoils", Journal of Micromechanics and Microengineering, vol. 18, no. 9, article ID 095018, 2008. DOI: 10.1088/0960$1317 / 18 / 9 / 095018$

[14] I. Lope, J. Acero, and C. Carretero, "Analysis and optimization of the efficiency of induction heating applications with litz-wire planar and solenoidal coils", IEEE Transactions on Power Electronics, vol. 31, no. 7, pp. 5089-5101, 2016. DOI: 10.1109/TPEL.2015.2478075.

[15] H. C. Séran and P. Fergeau, "An optimized low-frequency three-axis search coil magnetometer for space research", Review of scientific instruments, vol. 76, no. 4, pp. 044502-1-044502-10, 2005. DOI: 10.1063/1.1884026.

[16] F. Pellitteri, V. Boscaino, A. O. Di Tommaso, R. Miceli, and G. Capponi, "Experimental test on a Contactless Power Transfer system", in Proc. of 2014 Ninth International Conference on Ecological Vehicles and Renewable Energies (EVER), 2014, pp. 1-6. DOI: 10.1109/EVER.2014.6844092.

[17] S. Hasanzadeh, S. Vaez-Zadeh, and A. H. Isfahani, "Optimization of a Contactless Power Transfer system for electric vehicles", IEEE Transactions on Vehicular Technology, vol. 61, no. 8, pp. 3566-3573, 2012. DOI: 10.1109/tvt.2012.2209464.

[18] J. Sallan, J. L. Villa, A. Llombart, and J. F. Sanz, "Optimal design of ICPT systems applied to electric vehicle battery charge", IEEE Trans. Ind. Electron., vol. 56, no. 6, pp. 2140-2149, 2009. DOI: 10.1109/TIE.2009.2015359.

[19] J. Wang, M. Hu, Ch. Cai, Zh. Lin, L. Li, and Zh. Fang, "Optimization design of wireless charging system for autonomous robots based on magnetic resonance coupling", Aip Advances, vol. 8, no. 5, pp. 055004-1-055004-17, 2018. DOI: 10.1063/1.5030445.

[20] G. D Chavchanidze, Electrical Circuits of Sine Current. MIIT, Moscow, 2007.

[21] V. Kh. Pshikhopov and M. Yu. Medvedev, "Group control of autonomous robots motion in uncertain environment via unstable modes", SPIIRAS Proceedings, vol. 5, no. 60, pp. 39-63, 2018. DOI: 10.15622/sp.60.2.

[22] A. C. M. de Queiroz, "Mutual inductance and inductance calculations by Maxwell's Method", Home page of Dr. Antonio Carlos M. de Queiroz, 2005.

[23] H. A. Wheeler, "Simple inductance formulas for radio coils", Proceedings of the institute of Radio Engineers, vol. 16, no. 10, pp. 1398-1400, 1928.

[24] К. Д. Крестовников, А. Р. Шабанова, А. Д. Ковалёв, "Математическая модель роевой робототехнической системы с беспроводной двусторонней передачей энергии”, в Труды НИИР №1-2, 2020. DOI: 10.34832/NIIR.2020.1.1.007.

[25] K. Krestovnikov, E. Cherskikh, and A. Ronzhin, "Mathematical model of a swarm robotic system with wireless bi-directional energy transfer", in Robotics: Industry 4.0 Issues \& New Intelligent Control Paradigms. Studies in Systems, Decision and Control, vol. 272. 
Springer, Cham, 2020, pp. 13-23. DOI: 10.1007/978-3-030-378417_2.

[26] N. Pavliuk, A. Saveliev, E. Cherskikh, and D. Pykhov, "Formation of modular structures with mobile autonomous reconfigurable system", in Proc. of 14th International Conference on Electromechanics and Robotics "Zavalishin's Readings", 2019, pp. 383-395. DOI: 10.1007/978-981-13-9267-2_31.

[27] K. Krestovnikov, E. Cherskikh, and N. Pavliuk, "Concept of a synchronous rectifier for wireless power transfer system", in Proc. of IEEE EUROCON 2019 - 18th International Conference on Smart Technologies, 2019, pp. 1-5. DOI: 10.1109/eurocon.2019.8861856.

[28] K. Krestovnikov, A. Saveliev, A. Shabanova, and I. Vatamaniuk, "Comparative study of synchronous and non-synchronous rectifiers for use in the receiving part of a wireless charging system", in Proc. of 14th International Conference on Electromechanics and Robotics "Zavalishin's Readings", 2020, pp. 675-685. DOI: 10.1007/978-98113-9267-2_56.

[29] K. Krestovnikov, E. Cherskikh, and P. Smirnov, "Wireless power transmission system based on coreless coils for resource reallocation within robot group", in Proc. of International Conference on Interactive Collaborative Robotics, 2019, pp. 193-203. DOI: 10.1007/978-3-030-26118-4_19.

[30] K. D. Krestovnikov, E. O. Cherskikh, and A. R. Shabanova, "Circuit designs and engineering solutions based on synchronous rectifier for wireless energy transfer system", Modeling, Optimization and Information Technology, vol. 4, no. 27, 2019. DOI: 10.26102/23106018/2019.27.4.018

[31] I. V. Vatamaniuk and R. N. Yakovlev, "Algorithmic model of a distributed corporate notification system in context of a corporate cyber-physical system", Modeling, Optimization and Information Technology, vol. 7, no. 4, 2019. DOI: 10.26102/23106018/2019.27.4.026.

[32] I. V. Vatamaniuk and R. N. Yakovlev, "Generalized theoretical models of cyberphysical systems", Proceedings of the Southwest State University, vol. 23, no. 6, pp. 161-175, 2019. DOI: 10.21869/22231560-2019-23-6-161-175.

This article is an open access article distributed under the terms and conditions of the Creative Commons Attribution 4.0 (CC BY 4.0) license (http://creativecommons.org/licenses/by/4.0/). 